\title{
Protocolos de encaminamiento de datos para uso en microrredes rurales aisladas
}

\author{
Elvis E. Gaona*, Francisco Santamaría, Cesar L. Trujillo \\ Facultad Ingeniería, Universidad Distrital Francisco José de Caldas. Bogotá D.C - Colombia \\ (correo-e: egaona@udistrital.edu.co; fsantamariap@udistrital.edu.co; cltrujillo@udistrital.edu.co)
}

* Autor a quien debe ser dirigida la correspondencia

Recibido Oct. 1, 2019; Aceptado Dic. 3, 2019; Versión final Feb. 5, 2020, Publicado Jun. 2020

\begin{abstract}
Resumen
Este documento presenta los resultados obtenidos al comparar dos protocolos de encaminamiento reactivos: vector distancia bajo demanda ad hoc y el protocolo de fuente dinámica. Se evaluaron métricas de calidad de servicio en dos topologías de red inalámbricas en una microrred eléctrica rural aislada. La estructura de la red fue simulada en Network Simulator 2, comparando las métricas de calidad de servicio como lo son el retardo promedio, el caudal de datos, la tasa de paquetes entregados exitosamente y la variación del retardo para las topologías tipo de red: árbol y malla. Los resultados muestran que el protocolo de encaminamiento reactivo bajo demanda presentó mejores indicadores de calidad de servicio en ambas topologías. Se concluye que el protocolo de encaminamiento reactivo bajo demanda cumpliría con los requisitos de telecomunicaciones para la operación de una microrred rural aislada con baja densidad de nodos.
\end{abstract}

Palabras clave: microrred rural; encaminamiento reactivo; retardo promedio; topología de red

\section{Routing protocols for isolated rural micro-networks}

\begin{abstract}
This paper aims to present the results obtained by comparing two reactive routing protocols: ad hoc ondemand distance vector and dynamic source routing protocol. This served to evaluate quality of service quality metrics in two wireless network topologies in a rural isolated electric microgrid. The network infrastructure was simulated using the Network Simulator 2. The quality of service metrics compared in this study includes the average delay, the throughput, the rate of successfully delivered packets, and the jitter in two network topologies: tree and mesh. The results showed that the reactive routing on-demand protocol presented better service quality indicators in both topologies. In conclusion, the routing protocol complies with the communication requirements for the operation of a rural isolated microgrid with low node density.
\end{abstract}




\section{INTRODUCCION}

Las microrredes rurales se han desarrollado desde el principio, como un sistema de electrificación para las zonas de difícil acceso que no tienen la cobertura de las redes eléctricas tradicionales, debido a los elevados esfuerzos económicos y técnicos necesarios para su instalación (Schnitzer et al., 2014, Gómez et al., 2018). Están compuestas por elementos de generación de energía renovable y no renovable, cargas y sistemas de almacenamiento con capacidad de regulación propia, operando de forma aislada, en la mayoría de las situaciones, obteniendo así la máxima eficiencia del conjunto (Trujillo-Rodríguez et al., 2015; Bhoyar y Bharatkar, 2012; Cronje et al., 2012; Ding et al., 2013).

Para este propósito, se emplean redes de comunicaciones utilizadas para la transmisión de datos recolectados desde los nodos sensores ubicado en cada uno de los elementos de la microrred, requiriendo de nodos intermedios para llegar al nodo central de destino. Estos nodos hacen uso de algoritmos de encaminamiento para identificar la mejor ruta desde el nodo origen al nodo destino (Yerra et al., 2011), por esta razón se han desarrollado y clasificado varios algoritmos de encaminamiento para las redes de sensores inalámbricos según la estructura y funcionamiento (Al-Karaki y Kamal, 2004; Suarez-Chilma et al., 2018). Las redes de sensores inalámbricas han sido identificadas por el MIT (Massachusetts Institute of Technology) como una de las 10 tecnologías inalámbricas emergentes que han cambiado las comunicaciones en la última década (Van Der Werff, 2005), debido a que dan respuesta a las exigencias referentes al establecimiento de redes que cubren necesidades de enlace de forma flexible en tiempo, espacio, autonomía, autoconfiguración e independencia de una estructura fija (Capella, 2010).

Por otro lado, la integración de dispositivos de medición en microrredes eléctricas han requerido de diversos análisis, sin embargo, no se ha realizado un análisis que identifique la mejor opción aplicada a las necesidades de operación en microrredes eléctricas rurales aisladas. En este artículo se comparan los resultados obtenidos de dos protocolos de encaminamiento de datos aplicados a las condiciones de operación en una microrred rural aislada, operando con una infraestructura de comunicaciones con topología en árbol y malla. Las simulaciones realizadas muestran los tiempos de retardo, el caudal de datos, la tasa de paquetes entregados exitosamente y la variación del retardo (Calle et al., 2018), empleando algoritmos de encaminamiento propuestos por López-Mora y Rodríguez-Caballero (2015) con la herramienta NS2 (Network Simulator 2).

\section{OTROS ANTECEDENTES}

Hay una serie de antecedentes adicionales que es necesario detallar para documentar en mejor forma este trabajo: Microrredes aisladas de tipo rural y Protocolos de encaminamiento inalámbricos reactivos.

\section{Microrredes aisladas de tipo rural}

Las microrredes operan sobre una arquitectura de red eléctrica que les permite funcionar de forma autónoma a partir de diferentes configuraciones como lo son: el tipo y cantidad de generadores (renovables, no renovables), la forma de almacenamiento de energía (banco de baterías, celdas de combustible) y las características de la distribución eléctrica de los usuarios (residencial o industrial).

La selección del tipo de microrred (corriente continua o corriente alterna) a implementar, depende fuertemente de las tecnologías utilizadas en la generación y gestión de la energía, por ejemplo, la generación fotovoltaica y las baterías proporcionan corriente continua (CC), mientras que la generación con grupos electrógenos, eólica y pequeñas centrales hidroeléctricas proporcionan corriente alterna (CA) (Lasseter, 2002; Martínez-Cid y O'Neill-Carrillo, 2010). En zonas rurales se prefiere el uso de microrredes con acoplamiento mixto DC/CA donde la potencia suministrada por el inversor debe corresponder con la consumida por las cargas (González et al., 2017; Cronje et al., 2012; Schnitzer et al., 2014; Núñez Mata et al., 2013).

Estos dispositivos comparten información sobre la generación en las fuentes, consumo en las cargas y el estado de las baterías; que se recolectan a través de medidores inteligentes con capacidad de transferir datos de forma bidireccional, de esta manera se logra mantener actualizada la información sobre las pérdidas de sistema, las caídas de energía y facturación necesaria para realizar la gestión de los recursos energéticos. En la medida que crece y se expande la cantidad de medidores inteligentes, también aumenta la complejidad del sistema de comunicaciones (Carreño-Pérez, et al., 2019).

Dado que en la actualidad no existen algoritmos de encaminamiento aplicado a las redes de sensores inalámbricos en microrredes, aún se está estandarizando las capas de red y transporte del modelo ISO/OSI (Bukowski y Ranade, 2012; Gaona-García et al., 2014). Estos protocolos deben ser compatibles con las características de latencia según sus aplicaciones para microrredes mostradas en la Tabla 1. 
Tabla 1: Requerimientos de latencia en la operación de una red de comunicaciones sobre una microrred. (Gaona-García et al., 2014; Saputro et al., 2012)

\begin{tabular}{|l|l|}
\hline Aplicación & Latencia \\
\hline AMI (Advanced Meter Infrastructure) & $2-15 \mathrm{~s}$ \\
\hline Respuesta a la demanda & $500 \mathrm{~ms}$ - varios minutos \\
\hline Conocimiento del comportamiento en área amplia & $20-200 \mathrm{~ms}$ \\
\hline Recursos de energía distribuida y almacenamiento & $20 \mathrm{~ms}-15 \mathrm{~s}$ \\
\hline Transporte Eléctrico & $2 \mathrm{~s}-5 \mathrm{~min}$ \\
\hline Gestión Distribuida & $100 \mathrm{~ms}-2 \mathrm{~s}$ \\
\hline
\end{tabular}

\section{Protocolos de encaminamiento inalámbricos reactivos}

En entornos de las redes de sensores inalámbricos se han desarrollado algoritmos de encaminamiento con diferentes categorías para Smart Grid (Saputro et al., 2012), facilitando la implementación de dispositivos de medición de bajo costo y elevada duración, capaces de obtener información del entorno en una microrred, como la potencia generada por las fuentes de generación distribuida, el estado de las baterías y el consumo en las cargas. Estos datos son enviados de forma inalámbrica al nodo coordinador o concentrador para su posterior análisis y de esta forma realizar la gestión de los recursos energéticos (Castañeda et al., 2015; Capella , 2010). La transferencia de los datos en tiempo real desde los nodos sensores hacia el nodo concentrador y viceversa, requieren del establecimiento de rutas que deben calcularse cuando son demandadas, es decir, las rutas se descubren y se establecen bajo peticiones al momento de iniciar el enlace. Para este tipo de protocolos llamados bajo demanda se logran optimizar los recursos en la red de comunicaciones evitando el envío de paquetes de forma innecesaria, sin embargo, debe contemplarse el tiempo empleado al realizar el descubrimiento de una ruta (Li et al., 2010).

\section{Protocolo DSR (Dynamic Source Routing)}

Este protocolo se basa en el concepto de encaminamiento en origen, así los nodos mantienen cachés, donde las entradas incluyen el destino y la lista de nodos (saltos) para llegar a él (Ben et al., 2011).

\section{Protocolo AODV (Ad Hoc On Demand Distance Vector)}

Se mantiene la idea de los números de secuencia y tablas de encaminamiento, añadiendo el concepto de encaminamiento bajo demanda, es decir, que se establecen rutas con los nodos que intervienen en la comunicación (solo se guarda la información de los nodos seleccionados en la transmisión de datos). Este protocolo minimiza el tiempo de procesamiento, el consumo de memoria, el tráfico de control por la red. Adicionalmente, éste protocolo posee una tabla de encaminamiento por vector distancia de fácil implementación (Gaona-García, Mancera Lagos y Trujillo Rodríguez, 2016). Por tratarse de un protocolo bajo demanda el consumo de energía es bajo (Ben et al., 2011).

\section{METODOLOGÍA}

Los escenarios de simulación contemplan la topología de red: árbol y malla, asemejándose a las condiciones de conexión de los dispositivos de medición de energía en una microrred con una distribución de nodos con separación de 30 metros, ubicando al nodo cero como nodo coordinador de la red PAN en un área de cobertura de 250x250 metros. En la Figura 1 se muestran las dos topologías de red de área personal a simular. El sistema eléctrico a monitorear es trifásico, por lo que los datos recolectados utilizan 12 variables eléctricas (tensión, corriente, frecuencia y fase) con un tamaño de 1 byte para cada variable, resultando un tamaño de paquete de 100 bytes. En la Tabla 2 se muestra el resumen de los parámetros configurados en el simulador.

Para los protocolos de encaminamiento utilizados, se utilizaron modelos de encolamiento usados en la red inalámbrica relacionados con el tipo de algoritmo de encaminamiento utilizado, para el caso del protocolo AODV se utiliza el modelo Drop Tail, mientras que para el protocolo DSR se utiliza el modelo CMU PriQueue. Estos dos modelos de encolamiento se basan en el mecanismo FIFO (First In First Out), donde los paquetes son guardados en el orden que llegan y de igual manera son enviados en el mismo orden (Vivian, Alchieri y Westphall, 2006). En cuanto a la capacidad máxima del buffer se toma el valor de 150 bytes, utilizado comúnmente para estos dos modelos de encolamiento (Ben-Chikha et al., 2011; Sivakumar y Jaiswal, 2014). Finalmente, para hacer más reales las condiciones de simulación, se utilizan las características técnicas del módulo Transmisor/Receptor XBee Pro Serie 2 de uso comercial y bajo costo (Informatik y Bunyai, 2012), considerando en cada uno, los valores de potencia transmitida de $18 \mathrm{dBm}$ y umbrales de recepción en los nodos de $-103 \mathrm{dBm}$ para un tiempo de simulación de 100 segundos 

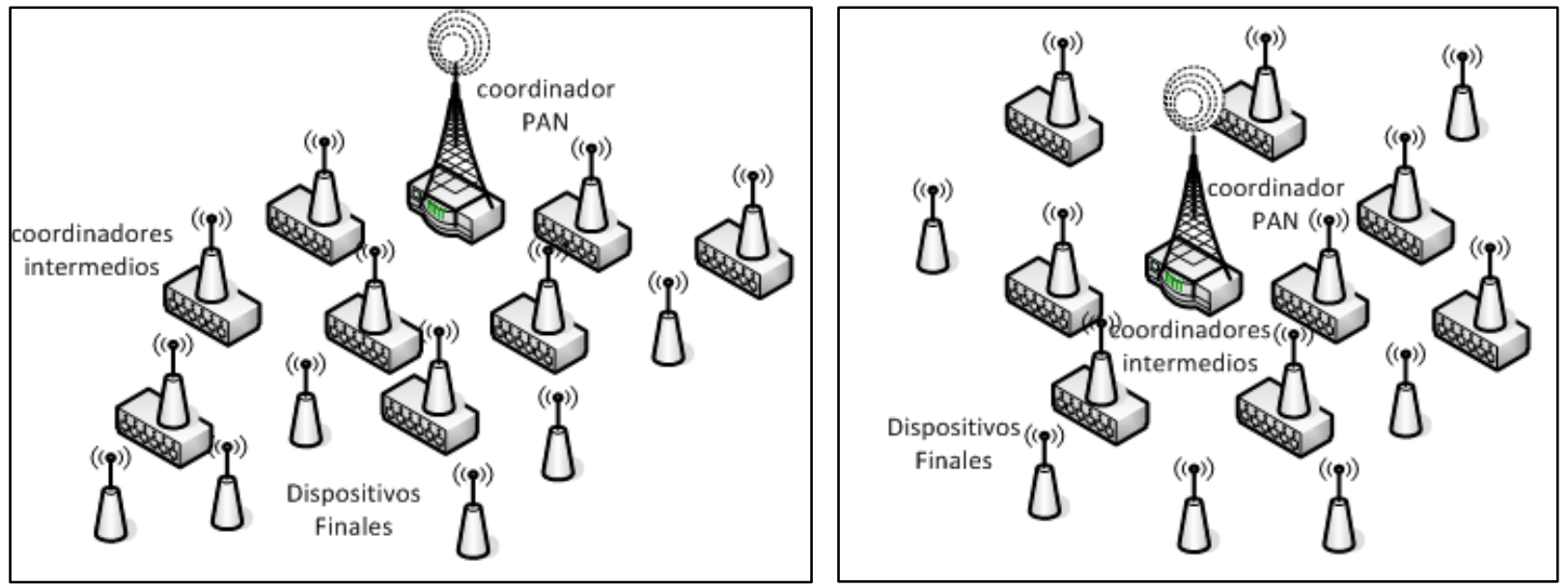

Fig. 1: Red PAN en topología árbol (izquierda) y malla (derecha).

Tabla 2: Tamaño en bytes de las variables medidas en los nodos sensores.

\begin{tabular}{|l|l|}
\hline Norma & ZigBee \\
\hline Protocolo encaminamiento & AODV \\
\hline Capas MAC/PHY & 802.15 .4 \\
\hline Topología de Red & Malla/Cluster \\
\hline Tipo de canal & Canal inalámbrico \\
\hline Modelo de propagación & Two Ray Ground \\
\hline Superficie (m) & 250 *250 \\
\hline Distancia entre nodos $(\mathrm{m})$ & 30 \\
\hline Numero de nodos & 7 a 31 \\
\hline IFQ (Interface queue type) & DropTail \\
\hline Data Payload (bytes/packet) & 100 \\
\hline Modelo Antena & Omnidireccional \\
\hline Potencia Rx (dBm) & -103 \\
\hline Potencia Tx (dBm) & 18 \\
\hline Tiempo simulación & $100 \mathrm{~s}$ \\
\hline
\end{tabular}

\section{RESULTADOS}

En el análisis comparativo de la red en topología árbol y malla se utilizaron las métricas de calidad de servicio como lo son: el caudal de datos, el retardo promedio, la relación de paquetes y la variación del retardo, estos parámetros permiten evaluar la estabilidad en la transmisión y recepción de los datos. En ese sentido, se realizaron dos grupos de simulaciones para las dos topologías, donde los nodos se ubicaron con 30 metros de separación entre ellos.

La Figura 2 muestra el comportamiento del caudal de datos y el retardo promedio (latencia) para diferente cantidad de nodos conectados en topología de red tipo árbol. Por un lado, el protocolo DSR presenta un mejor caudal de datos que el protocolo AODV para la zona de baja densidad de nodos, sin embargo, la curva disminuye drásticamente a medida que aumenta la densidad de nodos, esto se debe a la pérdida y rechazo de paquetes que no logran llegar a su destino final, perdiéndose el volumen de datos que la microrred genera desde los nodos sensores.

Por otro lado, el retardo promedio de transmisión de los datos es levemente menor en el protocolo DSR que con el protocolo AODV en la región de menor densidad de nodos (menor cantidad de nodos), debido a que el retardo se mide sobre los paquetes entregados satisfactoriamente, y se incrementa al aumentar cantidad de envíos exitosos. En contraste, el protocolo AODV mantiene un mejor caudal que el protocolo SDR con un promedio de $1.47 \mathrm{kbps}$ en comparación que el protocolo SDR con apenas $0.97 \mathrm{kbps}$. Esto se refleja directamente en el retardo promedio del protocolo AODV con un promedio de $44.6 \mathrm{~ms}$, mientras que con el protocolo PDR es de $31.5 \mathrm{~ms}$, debido a que a mayor cantidad de nodos se necesitan generar mayor cantidad de mensajes de encabezado en la fase de descubrimiento de la red. 

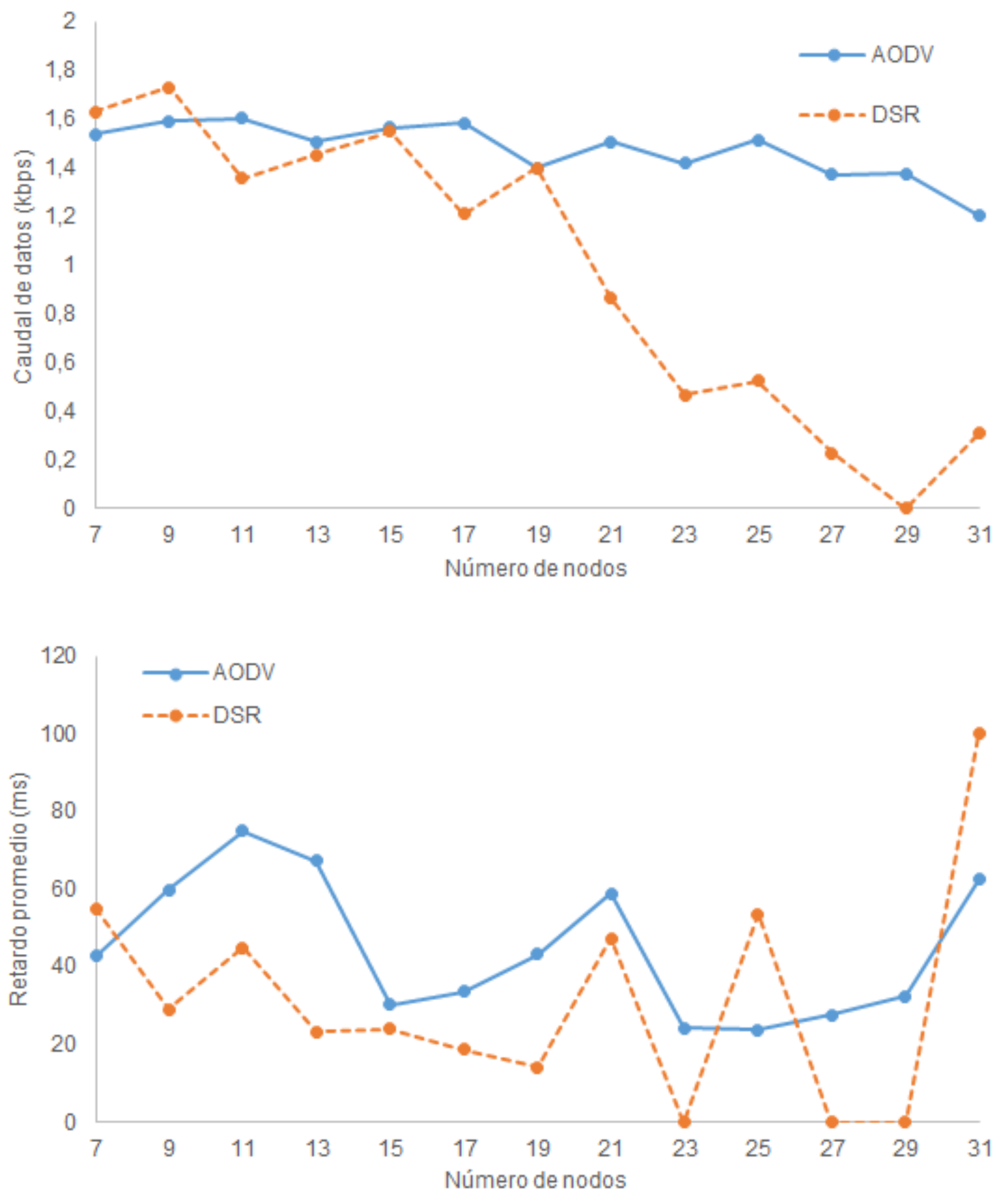

Fig 2: Caudal y retardo promedio de datos en topología árbol.

La Figura 3 muestra el comportamiento del caudal de datos y el retardo promedio de la red de sensores conectados ahora en topología malla versus el número de nodos. Las curvas del caudal de datos muestran que la ocupación del canal es mayor en comparación a la obtenida en la topología árbol, siendo mayor la diferencia entre las magnitudes del caudal de datos del protocolo AODV con un promedio de $10.36 \mathrm{kbps}$ y $4.23 \mathrm{kbps}$ para el protocolo DSR.

El caudal de datos en AODV aumenta con la densidad de nodos. El retardo promedio también aumento en ambos protocolos para esta topología en comparación con los resultados obtenidos para la topología árbol. El retardo promedio presentado para el protocolo AODV es en promedio $84.4 \mathrm{~ms}$, mientras que para el protocolo SDR es de $75.1 \mathrm{~ms}$, esto se debe a que existe una mayor cantidad de nodos coordinadores intermedios quienes envían constantemente mensajes de requerimiento de ruta (RREQ), generando un mayor flujo de datos en la red con un mayor tiempo para la recepción de los paquetes, ya que estos tienden a permanecer un mayor tiempo en los buffers de cola. 

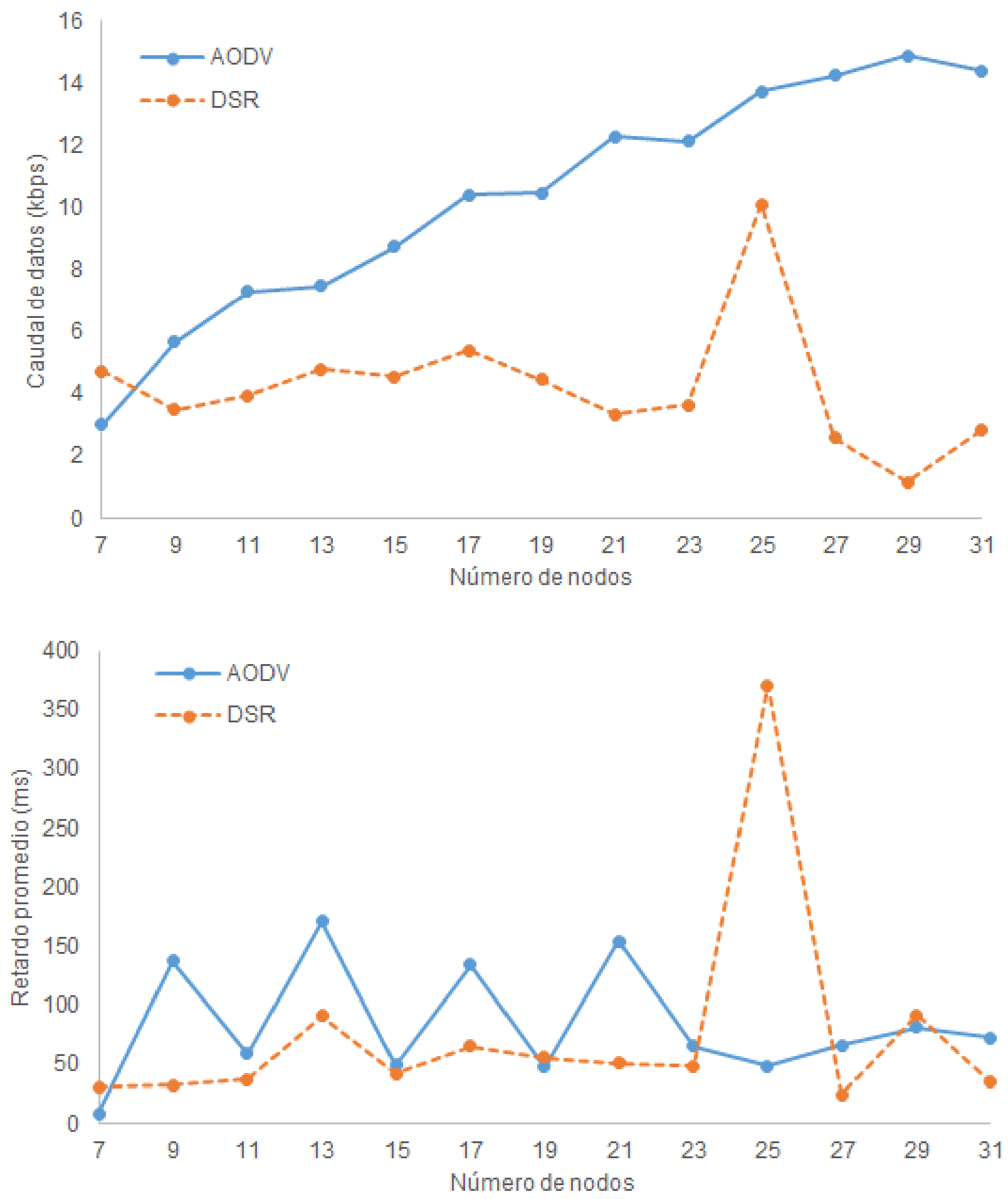

Fig. 3: Caudal de datos y retardo promedio en topología malla.

La Figura 4 muestra el comportamiento de la relación de paquetes entregados de forma exitosa y de la variación del retardo para una topología de red en árbol. Las curvas de la relación de paquetes entregados, muestran que es mayor usando el protocolo AODV, siendo en promedio de $96 \%$. Mientras que el protocolo SDR en promedio tiene una relación de apenas el $58 \%$ y disminuye drásticamente a partir de cierta cantidad de nodos (nodo 19). Esto evidencia que la pérdida de paquetes es mayor cuando se utiliza el protocolo DSR.

Estos resultados permiten verificar el comportamiento que se presenta con el retardo promedio. Con respecto a la métrica de la variación del retardo, los resultados muestran que existe una menor variación del retardo usando el protocolo AODV de apenas $5.66 \mathrm{~ms}$, representando mayor estabilidad en la red que cuando se utiliza el protocolo DSR. Aunque en la región de baja densidad de nodos el comportamiento de la variación del retardo es ligera, al aumentar la cantidad de nodos su comportamiento aumenta drásticamente, siendo de $154.68 \mathrm{~ms}$ para el protocolo SDR. 

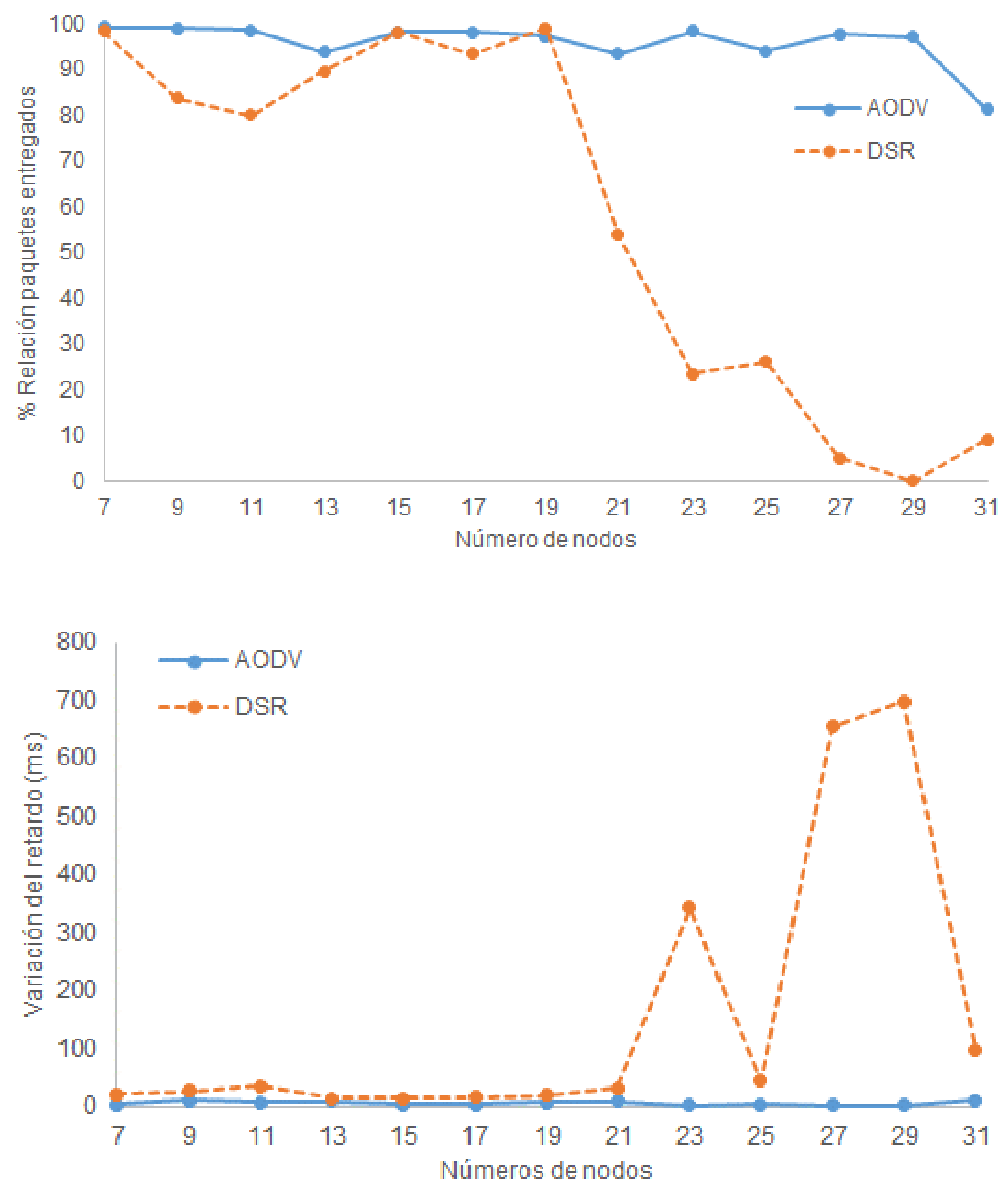

Fig. 4: Relación de paquetes entregados y la variación del retardo en topología árbol.

La Figura 5 muestra el comportamiento de las métricas de la relación de paquetes entregados exitosamente y la variación del retardo con la red en topología malla. Se puede observar que existe un menor porcentaje de entrega de paquetes al usar esta topología, en comparación a los resultados obtenidos para la misma métrica usando la topología de red en árbol, este comportamiento permite afirmar que existe una mayor pérdida de paquetes en la topología malla, aunque el protocolo AODV nuevamente presenta una menor perdida de paquetes frente al protocolo DSR. Para la zona de menor densidad de nodos el protocolo SDR presenta mejor relación de paquetes entregados que el protocolo AODV, sin embargo, al aumentar la cantidad de nodos, y la diferencia para la topología malla se evidencia en una mayor diferencia de la relación de paquetes entre ambos protocolos. Para la métrica de la variación del retardo de nuevo se observa que el protocolo AODV mantiene una mayor estabilidad de la red en comparación con el protocolo DSR. También se puede observar que la variación del retardo tiene una mayor magnitud en la topología malla frente a los resultados obtenidos en la topología árbol. 

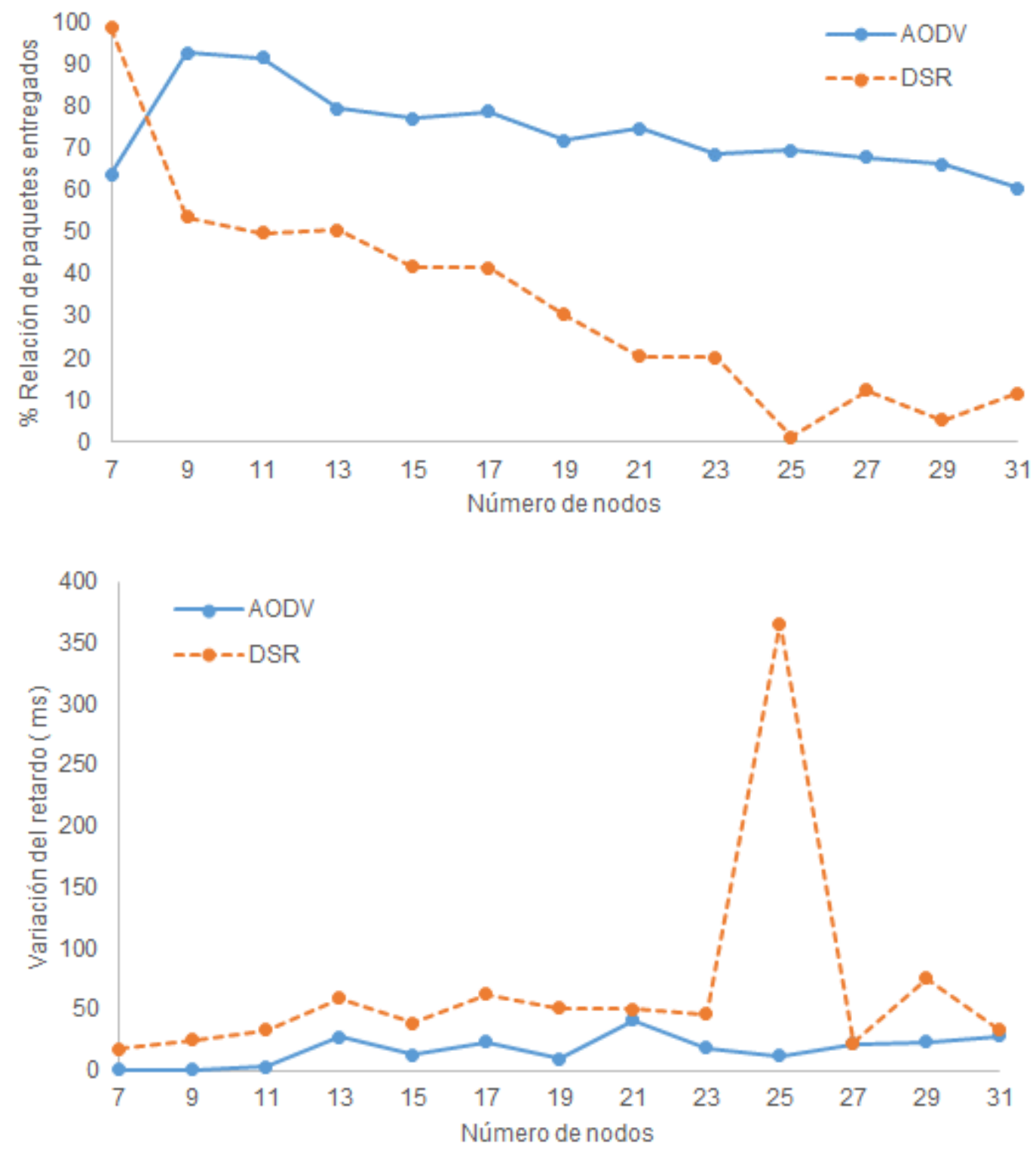

Fig. 5: Relación de paquetes y variación del retardo en topología malla.

\section{CONCLUSIONES}

A partir de los resultados obtenidos en las simulaciones se concluye que el protocolo de encaminamiento AODV presenta mejor desempeño para un modelo de red de comunicaciones en topología árbol, dado que tiene una menor perdida de paquetes, los tiempos de retardo promedio, la variación del retardo y el caudal de datos son bajos. Para la topología en malla, este protocolo es funcional solo para una baja densidad de nodos. Por otro lado, el protocolo de encaminamiento SDR cumple con los requerimientos de comunicaciones para la operación de una microrred rural aislada con topologías en árbol y malla para una baja densidad de nodos, mientras que, para una densidad de nodos alta, la topología en malla no es funcional debido a que tiene una tasa de entrega de paquetes que empeora al aumentar la cantidad de nodos. Finalmente, se evidenció que el uso del canal de comunicaciones en ambas topologías cumple con los requerimientos necesarios para la operación de una microrred rural que utilice una infraestructura de medición avanzada con una densidad de nodos, condición natural en ambientes rurales aislados.

\section{AGRADECIMIENTOS}

Esta investigación es financiada por el Centro de Investigación y Desarrollo Científico de la Universidad Distrital Francisco José de Caldas - CIDC (Proyectos códigos: 2-5-524-16 y 2-7-563-17).

\section{REFERENCIAS}

Al-Karaki, J.N. y Kamal A.E., Routing techniques in wireless sensor networks: a survey, https:doi.org/10.1109/MWC.2004.1368893, IEEE Wireless Communications, 11(6), 6-28 (2004) 
Ben Chikha, H., Makhlouf A. y Ghazel W., Performance analysis of AODV and DSR routing protocols for IEEE 802.15.4/ZigBee, https://doi.org/10.1109/CCCA.2011.6031459, 2011 International Conference on Communications, Computing and Control Applications, CCCA 2011, 4-8 (2011)

Bhoyar, R. y Bharatkar S., Potential of MicroSources, Renewable Energy sources and Application of Microgrids in Rural areas of Maharashtra State India, https://doi.org/10.1016/j.egypro.2011.12.1202, Energy Procedia, 14, 2012-2018 (2014)

Bukowski, S. y Ranade S., Communication network requirements for the Smart Grid and a path for an IP based protocol for customer driven microgrids, https://doi.org/10.1109/EnergyTech.2012.6304640, Energytech, 2012 IEEE, 1-6 (2012)

Calle, M.A., Tovar J.D., y otros 2 autores, Comparación de Parámetros para una Selección Apropiada de Herramientas de Simulación de Redes, https://doi.org/10.4067/S0718-07642018000600253, Información Tecnológica, 29(6), 253-266 (2018)

Capella-Hernández, J.V., Redes inalámbricas de sensores: una nueva arquitectura eficiente y robusta basada en jerarquía dinámica de grupos, $1^{\text {a }}$ Ed., Universidad Politécnica de Valencia, Valencia, España, (2010)

Carreño-Pérez, J.C., Morales-Rivera J.P., y Rivas-Trujillo E., Redundancia en Redes de Comunicación para la Automatización y Protección de Sistemas de Potencia Eléctrica con IEC 61850, https://doi.org/10.4067/S071807642019000100075, Información Tecnológica, 30(1), 75-86 (2019)

Castañeda Fandiño, I., Gaona García E., y Trujillo Rodríguez C., Proceso de estimación estocástico para la selección de fuentes de energía en una microrred eléctrica, https://doi.org/10.14483/22487638.9620, Tecnura, 19(CITIE), 137-144 (2015)

Cronje, W.A., Hofsajer I.W., y Braid J.I., Design Considerations for Rural Modular Microgrids, 743-748, (2012)

Ding, Z., Liu M., y otros 2 autores, An autonomous operation microgrid for rural electrification, https://doi.org/10.1109/IAS.2013.6682482, 2013 IEEE Industry Applications Society Annual Meeting, 1-8 (2013)

Gaona-García, E. E., Mancera Lagos P.A., y Trujillo Rodríguez C.L., Algoritmo de encaminamiento con reconfiguración de topología para red de sensores inalámbricos aplicada a una Microrred en modo "Isla", https://doi.org/10.18273/revuin.v15n2-2016008, UIS Ingenierías, 15(2), 93-104 (2016)

Gaona-García, E.E., Trujillo-Rodríguez C.L. y Rojas Cubides H.E., Infraestructura de comunicaciones en microrredes eléctricas, https://doi.org/10.14483/2248762X.8044, Redes de Ingeniería, 5(2), 28-38 (2014)

Gómez, V. A., Hernández C., y Rivas E., Visión General, Características y Funcionalidades de la Red Eléctrica Inteligente (Smart Grid), https://doi.org/10.4067/S0718-07642018000200089, Información Tecnológica, 29(2), 89-102 (2018)

González, N., Cusgüen C., y otros 2 autores, Estrategias de control de calidad de energía en microrredes rurales, https://doi.org/10.18273/revuin.v16n2-2017009, Revista UIS Ingenierías, 16(2), 93-104 (2017)

Informatik, T. y Bunyai D., ZigBee Network Layer Simulation, (2012)

Lasseter, R. H., MicroGrids, https://doi.org/10.1109/PESW.2002.985003, 2002 IEEE Power Engineering Society Winter Meeting. Conference Proceedings (Cat. No.02CH37309), 1, 305-308 (2002)

$\mathrm{Li}$, J., Zhu X. y otros 2 autores, Study on ZigBee network architecture and routing algorithm, https://doi.org/10.1109/ICSPS.2010.5555486, ICSPS 2010 - Proceedings of the 2010 2nd International Conference on Signal Processing Systems, 2(c), 389-393 (2010)

López-Mora, D. E. y Rodríguez-Caballero W.D., Acondicionamiento de un algoritmo de encaminamiento al sistema de comunicaciones en una microrred. Universidad Distrital Francisco José de Caldas (2015)

Martínez-Cid, R. y O'Neill-Carrillo E., Sustainable microgrids for isolated systems, https://doi.org/10.1109/TDC.2010.5484216, 2010 IEEE PES Transmission and Distribution Conference and Exposition: Smart Solutions for a Changing World, 1-7 (2010)

Núñez-Mata, O., Ortiz-villalba D. y Palma-Behnke R., Microrredes en la red eléctrica del futuro - caso huatacondo, ISSN: 0378-0524, Ciencia y Tecnología, 29(2), 1-16 (2013)

Saputro, N., Akkaya K. y Uludag S., A survey of routing protocols for smart grid communications, doi: 10.1016/j.comnet.2012.03.027, Computer Networks, 56(11), 2742-2771 (2012)

Schnitzer, D., Deepa S. y otros 4 autores, Microgrids for Rural Electrification : A critical review of best practices based on seven case studies Microgrids for Rural Electrification : A critical review of best practices. United Nations Foundation, 122, (2014)

Sivakumar, N. y Jaiswal S.K., Comparison of DYMO protocol with respect to various quantitative performance metrics. Department of Computer Science, Malardalen University (2014)

Suarez-Chilma, V.F., Sarache W.A. y Costa Y.J., Una Solución al Enrutamiento de Vehículos en Ciudades Montañosas Considerando Aspectos Ambientales y Económicos, https://doi.org/10.4067/S0718-07642018000300003, Información Tecnológica, 29(3), 3-14 (2018)

Trujillo Rodríguez, C.L., Santamaría Piedrahita F. y otros 8 autores, Generalidades de Microrredes Eléctricas. Microrredes Eléctricas, $1^{\text {a }}$ Ed., Universidad Distrital Francisco José de Caldas, 13-27, Bogotá D.C, Colombia (2015) 
Van Der Werff, 10 Emerging Technologies That Will Change the World, from http://www2.technologyreview.com/featuredstory/401775/10-emerging-technologies-that-will-change-the/2/, (2005)

Vivian, D., Alchieri E.A.P. y Westphall C.B., Evaluation of QoS Metrics in Ad Hoc Networks with the use of Secure Routing Protocols, https://doi.org/10.1109/NOMS.2006.1687606, 2006 IEEE/IFIP Network Operations and Management Symposium NOMS 2006, 1-4 (2006)

Yerra, R.V.P., Bharathi A.K. y otros 2 autores, WSN based power monitoring in smart grids, https://doi.org/ 10.1109/ISSNIP.2011.6146589, Intelligent Sensors, Sensor Networks and Information Processing (ISSNIP), 2011

Seventh International Conference on Intelligent Sensors, Sensor Networks and Information Processing, 401-406 (2011) 